\title{
Herd-level prevalence of the ultrasonographic lung lesions associated with bovine respiratory disease and related environmental risk factors
}

\author{
S. Buczinski, ${ }^{1}$ M. E. Borris, ${ }^{2}$ and J. Dubuc \\ Département des Sciences Cliniques, Faculté de Médecine Vétérinaire, Université de Montréal, Saint-Hyacinthe J2S 2M2, Québec, Canada
}

\section{ABSTRACT}

Bovine respiratory disease complex (BRD) is a major calf disease during the preweaning period. Thoracic ultrasound (TUS) has been recently described as a reliable tool for assessing BRD-associated lung lesions. The objectives of this study were to define the herdlevel prevalence of lung consolidation assessed by TUS (CONSTUS). A total of 39 Québec dairy herds were randomly chosen to participate in this cross-sectional study. Between 6 and 12 preweaned calves were examined for signs of CONSTUS defined by any site with $\geq 3$ $\mathrm{cm}$ consolidated lung tissue during 1 visit in summer and 1 visit in winter. Herd questionnaire focused on calf health and housing data [airborne bacteria (aerobic, coliform, yeasts and mold counts), air drafts, temperature, hygrometry, and ammonia levels] were also collected during these visits looking for potential association with CONSTUS prevalence. The median herd-level of CONSTUS prevalence (interquartile range) were $8 \%(0-22 \%)$ in summer and $15 \%(0-35 \%)$ in winter. Multivariable analyses showed that season was associated with an increased CONSTUS prevalence [10.0 vs. $19.3 \%$; odds ratio $(\mathrm{OR})=2.16]$, as well as group housing during preweaned period (9.6 vs. $20.0 \%$; OR $=$ $2.37)$ and perceived BRD problem by the farmer (10.6 vs. $18.3 \%$; OR $=1.89$ ). Despite tremendous changes in calves' environment between winter and summer, none of the housing variables were associated with the CONSTUS prevalence in this study. Based on the observed CONSTUS prevalence ranges in the present study (herds in the lower prevalence quartile), an achievable goal of none of 12 calves with consolidation $\geq 3 \mathrm{~cm}$ can potentially be used to define a low-BRD risk herd.

Key words: enzootic pneumonia, preweaning, imaging, diagnosis

\footnotetext{
Received July 7, 2017.

Accepted November 11, 2017.

${ }^{1}$ Corresponding author: s.buczinski@umontreal.ca

${ }^{2}$ Current address: Vankleek Hill Veterinary Services, Vankleek Hill, Ontario, Canada.
}

\section{INTRODUCTION}

Bovine respiratory disease complex (BRD) is a multifactorial disease resulting from interaction between the host, the microbiological agents, and environmental factors. In dairy calves, enzootic pneumonia is classically used to depict the typical pattern of occurrence of several cases that regularly occur (Van Donkersgoed et al., 1993). Besides the specific effect related to morbidity and mortality costs, BRD also has a negative effect on growth and long-term productivity (Guterbock, 2014). Predisposing factors of BRD include inadequate transfer of passive immunity (Van Donkersgoed et al., 1993; Furman-Fratczak et al., 2011; Windeyer et al., 2014), inadequate vaccination protocols (Gorden and Plummer, 2010), as well as diet restriction (e.g., ingestion of low milk amounts) which can depress immune function (Khan et al., 2011). The environmental factors commonly reported to be associated with greater risk of BRD include group housing (Svensson and Liberg, 2006), poor bedding quality (Lago et al., 2006), large group size (Svensson and Liberg, 2006), direct contact with older animals (Lundborg et al., 2005), and poor microbiological air quality (Lago et al., 2006).

The clinical signs of BRD can be insidious (McGuirk and Peek, 2014); although helpful, clinical signs are not perfectly accurate to diagnose BRD (Ollivett et al., 2015). The clinical presentation of the disease may be difficult to diagnose correctly despite the existence of several screening strategies based on clinical scoring (McGuirk, 2008; Aly et al., 2014). Thus, thoracic ultrasound (TUS) has been recently mentioned as an interesting diagnostic tool that could be implemented on farms for a rapid scanning of dairy calves' lungs (Ollivett and Buczinski, 2016). Observation of pleural and lung anomalies can be used as a proxy for diagnosis of BRD with a higher sensitivity and specificity than clinical scoring (Buczinski et al., 2015) and a good correlation with necropsy findings (Ollivett et al., 2015). Moreover, extensive lung lesions assessed by TUS at weaning are associated with an increased risk of being prematurely culled before the first calving (Adams and Buczinski, 2016; Teixeira et al., 2017). In a randomized 
controlled trial assessing the efficacy of intranasal vaccination in dairy calves, animals that were found with at least 1 lesion $\geq 3 \mathrm{~cm}$ of lung consolidation depth had $0.1 \mathrm{lbs} / \mathrm{d}(45.4 \mathrm{~g} / \mathrm{d})$ less of daily weight gain than nonconsolidated calves over a time period of $56 \mathrm{~d}$ (Ollivett, 2014). It would therefore be of interest to gather more data on ultrasonographic lesions in preweaning dairy calves as a way to assess calf health and productivity.

Unfortunately, data quantifying calf- and herdlevel prevalence of lung consolidation assessed by TUS (CONSTUS) are scant. In a previous study performed in 13 Québec dairy farms (Buczinski et al., 2014), 56 of 106 calves $(53 \%)$ had ultrasonographic evidence of lung consolidation (at least $1 \mathrm{~cm}$ of consolidated lung). At the herd level, the prevalence of consolidation in this previous study varied from 0 to $78 \%$ (median 50\%; interquartile range $50-60 \%$ ). However, this study was performed on a small number of herds obtained by convenience sampling and focused at enrolling herds with enzootic BRD problems; therefore, the findings cannot be extrapolated to the general population of dairy farms. For these reasons, it is difficult to know the prevalence of CONSTUS in preweaning dairy calves at the herd level. Knowing the latter information as well as potential herd-level risk factors associated with such prevalence could be valuable to assess respiratory health in preweaning calves of dairy farms.

Poor air quality and ventilation have classically been described as a risk factor for BRD in dairy calves at the herd level (Pritchard et al., 1981; Lago et al., 2006). The assessment of air quality can be performed in multiple ways (Lago et al., 2006). Air drafts as well as ammonia or airborne microbiological flora have been associated with an increased risk of BRD at the herd level (Lundborg et al., 2005; Lago et al., 2006). In a previous study using an air-sampler for aerobic and coliform culture, the authors reported that aerobic counts, but not coliform counts, were associated with BRD prevalence in a naturally ventilated barn during the winter (Lago et al., 2006). Mold and fungi are also commonly found in livestock buildings (Blom et al., 1984) and have been associated with allergic respiratory diseases in horses or human (Beeler-Marfisi et al., 2010; Pomés et al., 2016). Recently, on-farm culture systems have been developed and adapted for a practical use to assess etiologic agents of cows' mastitis (Cameron et al., 2013). The Petrifilm culture system has also been validated for the assessment of airborne bacteria, mold, and yeasts and was recently described as a relatively inexpensive alternative to assess microbiological quality of farm environment (Buczinski et al., 2017).

Our hypotheses were that the prevalence of CONSTUS would be higher in winter than in summer and that it would be associated with the same herd risk factors as those associated with clinical BRD. The main objective of our study was to report the herdlevel prevalence of CONSTUS in dairy calves from a large number of herds. A secondary objective was to describe environmental or herd factors associated with the proportion of consolidated calves on farms.

\section{MATERIALS AND METHODS}

A cross-sectional study was performed at the bovine ambulatory clinic of the Faculté de médecine vétérinaire of the Université de Montréal (St-Hyacinthe, QC, Canada) during 2 time periods, July to August 2015 (summer) and December 2015 to January 2016 (winter). The herd was the unit of interest in this study. Herd selection was randomly performed within all the herds using veterinary services from the bovine ambulatory clinic $(\mathrm{n}=161$; source population). For this purpose, a list of all the herds from the clinic was built. For practical considerations, all herds with limited size $(<50$ lactating cows) were excluded (not having enough calves to examine within a 2 -mo period). The random function of Microsoft Excel (Microsoft Corp., Redmond, WA) was used to select herd from that list. Selected dairy producers were then asked to confirm their participation through an individual phone call.

\section{Sample Size Calculation}

The herd-level sample size estimation (i.e., herd number needed) for the main objective (prevalence) was estimated to be 32 herds and was based on the following assumptions: prevalence expected $=10 \%, \alpha$ $=5 \%$ (for a $95 \%$ CI), and precision of estimate $=7.5 \%$. This 10\% prevalence was based on our best guess and a study in male dairy calves $(\mathrm{n}=209)$ with low BRD risk systematically screened with ultrasound at 2 to 3 wk of age, where prevalence of lung lesion was 12\% (Berman et al., 2017). The herd-level sample size estimation for the secondary objective (risk factors) was estimated to be 40 herds and was based on finding a difference of $35 \%$ in prevalence (absence of risk factor $=5 \%$; presence of risk factor $=40 \%$ ), and accounting for $\alpha$ and $\beta$ errors of 5 and $20 \%$, respectively. Therefore, recruitment of 40 herds was targeted. We were able to recruit a total of 39 herds for the study, which represented a $24 \%$ participation rate from the source population. In each participating herd, 6 to 12 preweaning calves were targeted to be sampled during a farm visit. This within-herd sample size calculation was based the following assumptions: prevalence expected $=5 \%, \alpha=$ $25 \%$ (for a $75 \% \mathrm{CI}$ ), and precision of estimate $=10 \%$. 


\section{Assessment of Herds and Calf Rearing Characteristics}

During each study period, every herd was visited once by a veterinarian and a research technician for data collection. Specific information concerning calf management during the preweaning period was collected from the producer during the farm visit. The average numbers of milking cows during the sampling periods were obtained, as well as vaccination protocols used for the adults cows and for the preweaning calves. The total number of preweaning calves at the day of the visit was also recorded. If more than 12 preweaning calves were present at the time of the visit, the sampling among available calves was performed taking into account the weighted proportion of calves housed in similar pen or housing conditions to be representative of herd structure at this time. Specific information collected concerning the preweaning period were the nature of the liquid diet [milk replacer (MR) vs. waste milk], the maximal daily volume received by a calf during this period, as well as the criteria for weaning (age, weight, or both). The feeding technique (individual feeding such as bottle, bucket with or without nipple vs. automatic milk feeder) and the presence of free access to water for all calves were noted. The main bedding component and the nesting score ( 1 to 3 scale) in up to 5 calves were recorded as previously described (Lago et al., 2006). The calves that were lying down during the visit were scored and an average score was obtained by dividing the sum of the scores by the number of calves assessed. The type of contact with other animals was also quantified. Contact with other animals was considered as absent or minor if calves were raised individually and had a contact limited to their penmates. The contact was considered significant if animals were group-raised or had direct contact with other animals. The age of the animals that were in contact with preweaning calves was also taken into account. The farms were classified as having preweaning calves with no direct contact with other animals or preweaning calves with direct contact with animals of their age or direct or indirect contact with other animals (postweaning calves, heifers, or cows). The owners were also asked at the day of the visit if they had any concern on current pneumonia problems in preweaning calves.

\section{Assessment of Microbiological Air Quality}

The assessment of airborne bacteria, yeasts, and mold counts was performed using on farm culturing systems (Petrifilms, 3M Canada, London, ON, Canada). Three different types of plates were used.
The total aerobic count plate (TAC), coliform count (CC), and yeasts and mold plates (YM) were used to assess the microbiological air quality in the barn, as previously reported (Buczinski et al., 2017). The plates were used according to the manufacturer specification for air sampling, incubation, and reading. The plates were rehydrated prior farm visit with $1 \mathrm{~mL}$ of sterile water solution and kept refrigerated up to $7 \mathrm{~d}$. As the study objective was to focus on calves' environment, Petrifilm samples were only taken at the calf housing site. The site of air quality assessment needed to be representative of typical calf environment on that particular farm. In the case of external hutches, only 1 random hutch was sampled. In the case of calves raised attached in the front or back of the cows, because we found no difference between calf environment and feeding alley, only 1 sample was taken at the calf site. In the case of individual pens, 1 occupied pen was randomly sampled. When calves were raised in groups with automatic milk feeders, sampling was performed in the pen approximatively $2 \mathrm{~m}$ far from the milk line. The samples at the calf site were always taken in occupied pens, boxes, or hutches. To avoid any movement of the calf that could contaminate Petrifilm plates, the calves were attached and moved away from the individual pen or hutch during the sampling period. One operator was present in the pen of group-raised calves to limit risk of contamination due to calf movements. The samples were always taken at 20 to $30 \mathrm{~cm}$ from the ground to be closely associated with what a calf breathes when being down. The system used was a roof plastic base (Oatey, Cleveland, $\mathrm{OH}$ ), where the plates were kept in place using metal binders avoiding any contact with growth medium. The Petrifilm manufacturer recommends air contact up to $15 \mathrm{~min}$ to assess airborne bacteria in factories. However, based on a previous study, we used a 10-min sampling period, which was considered as a good compromise contact time (Buczinski et al., 2017). Contact time assessment was assessed using chronometer function included in a smartphone (iPhone 5, Apple, Cupertino, CA).

The Petrifilm plates were incubated (Boeker digital incubator, Boeker Scientific, Feasterville, PA) and read according to the manufacturer recommendations (https://www.3mcanada.ca/3m/en_ca/food-safety -testing-ca/products?Ntt=petrifilm\&LC=en_CA\&co $=$ cc\&gsaAction $=$ scBR\&type $=c c)$. Briefly, the aerobic count was read $48 \mathrm{~h}$ after incubation and the coliform plate $24 \mathrm{~h}$ after incubation at $35^{\circ} \mathrm{C}$. The yeast and mold plate were read 3 to $5 \mathrm{~d}$ after incubation at room temperature $\left(20-25^{\circ} \mathrm{C}\right)$. In case the plate count exceeds 1,000 (i.e., too numerous to be counted), the value of 1,001 was given. 


\section{Assessment of Air Draft, Temperature, Hygrometry, and Ammonia}

The sites that were used for microbiological sampling were also used for air draft assessment with a portative anemometer (Velocicalc 9565, TSI Inc., Shoreview, $\mathrm{MN})$ assessing wind speed $(0-50 \mathrm{~m} / \mathrm{s}$; accuracy \pm 0.015 $\mathrm{m} / \mathrm{s})$, relative hygrometry $(0-95 \%$ relative humidity, accuracy $\pm 3 \%$ ), and temperature $\left(-10\right.$ to $60^{\circ} \mathrm{C}$, accuracy $\pm 0.3^{\circ} \mathrm{C}$ ). The sampling period was $2 \mathrm{~min}$ in duration, with the probe oriented to the ventilation system at the 20 to $30 \mathrm{~cm}$ from the ground. The recordings were stored in a laptop and minimal, maximal, and mean values were kept for further analysis. The ammonia level was also recorded at the same sites using a handheld ammonia analyzer (GasAlertNH3, Honeywell Analytics, Lincolnshire, IL) with a range detection of 0 to $50 \mathrm{ppm}$. An increased ammonia level was considered if ammonia concentration was $\geq 5 \mathrm{ppm}$ to take into account recent recommendations (between 3.5 and 7 ppm) in Canadian dairy farms (Kaufman et al., 2015).

\section{Calf Data Measurements}

During the farm visits, each calf was examined and a systematic thoracic ultrasonography was performed for screening 3 different lung areas: the right and left lung area caudal to the heart, and the right cranial part of the lung (Ollivett and Buczinski, 2016). Alcohol was sprayed on the intercostal space and the area of interest was screened using a linear probe (Draminski iScan, Olsztyn, Poland), focusing on any area of consolidation in particular. The maximal depth of consolidation $(\mathrm{cm})$ was noted for each calf at each site, as described elsewhere, using the $1-\mathrm{cm}^{2}$ grid on the screen (Buczinski et al., 2014). A calf was considered to have ultrasonographic evidence of consolidation if consolidation depth was $\geq 3 \mathrm{~cm}$, as that depth has been previously associated with decreased preweaning ADG (Ollivett, 2014).

\section{Statistical Analyses}

The statistical analyses were performed using commercial software such as SAS (version 9.4, SAS Institute Inc., Cary, NC) and MedCalc (version 13.1.0, Ostend, Belgium). Descriptive statistics concerning herd characteristics and prevalence of ultrasonographic consolidation were calculated using a depth of $3 \mathrm{~cm}$ as cutoff ( $<3$ vs. $\geq 3 \mathrm{~cm})$.

Variable Selection. The correlation between the different collected variables was performed using Spearman correlation (CORR procedure of SAS). Multicollinearity was considered for a Spearman correlation $\geq 0.7$, and only 1 variable was kept for the analysis. For continuous data (bacterial and yeasts and mold counts), normality was assessed using Shapiro Wilk test in UNIVARIATE procedure of SAS. As the data were right-skewed, a natural logarithm transformation was made to improve data normality for TAC, CC, and YM counts before using them for univariable analysis. The association between the proportion of calves with lung lesions and calf age in weeks (from wk 1 to $\geq 9$ ) was done using multiple comparisons test for proportion with Tukey correction for multiple comparisons (\% COMPPROP macro of SAS). The median age of sampled calves in every herd was added as a potential covariable that could affect the prevalence of $\mathrm{CON}$ STUS.

Herd-Level Models. The season was considered as a fixed effect and forced in the models. The within-herd prevalence of consolidated calves was assessed using a mixed logistic regression model using the GLIMMIX procedure of SAS (event/trial syntax, logit link). The farm was considered as the cluster of replication during both seasons [1]. The general linear mixed model was described as (Stroup, 2012)

$$
\text { Logit } \pi=\boldsymbol{\beta} \mathbf{X}+\mathbf{F b} \text {, }
$$

where $\pi$ is the prevalence of calves with consolidation (calves consolidated/calves screened) in the ith farm; $\boldsymbol{\beta}$ is the vector of model parameters; $\mathbf{X}$ is a matrix of farm covariates that also include season as a fixed effect; the term $\mathrm{b}\left(0 ;{\sigma_{\mathrm{F}}}^{2}\right)$ is the random effect for farm vector $\mathbf{F}$.

Univariable analyses were performed (all the different tested variables are summarized in Tables 1 and 2 ). Categorical variables were offered specifying the CLASS statement and continuous transformed variables (log-transformed colony-forming unit plates and median age of calves per herd) were assessed using the same univariable mixed model taking into account farm clustering (GLIMMIX procedure). The variables with $P$ $<0.2$ using an F-test were offered to the multivariable model using a backward elimination strategy until all remaining variable had $P$-values $<0.05$. A 2 -way interaction term (season $\times$ Petrifilm microbiological count) was also tested, as it was anticipated that season could potentially interact with microbiologic air quality. A question was asked to the farmers about their perception of having a BRD problem at the time of the farm visits. This information was used as a potential variable that could be positively associated with the proportion of consolidated calves. We also built a model without including this covariate.

Fit statistics for conditional distribution of the generalized linear mixed model was considered adequate 
Table 1. Descriptive statistics of environment characteristic in farms in winter and summer and univariable association with the prevalence of ultrasonographic consolidation ( $\geq 3 \mathrm{~cm}$ depth) in preweaned calves

\begin{tabular}{|c|c|c|c|c|c|c|c|c|}
\hline \multirow[b]{2}{*}{ Parameter } & \multicolumn{3}{|c|}{ Summer } & \multicolumn{3}{|c|}{ Winter } & \multirow{2}{*}{$\begin{array}{c}P \text {-value } \\
(\text { between season })^{1}\end{array}$} & \multirow{2}{*}{$\begin{array}{l}P \text {-value } \\
\text { (univar) }^{2}\end{array}$} \\
\hline & Mean & Median & Range & Mean & Median & Range & & \\
\hline Coliform count (cfu/plate) & 7 & 4 & $1-35$ & 2 & 1 & $0-18$ & $<0.01$ & 0.11 \\
\hline Yeasts count (cfu/plate) & 23 & 12 & $0-300$ & 33 & 17 & $1-310$ & 0.19 & 0.37 \\
\hline Mold count (cfu/plate) & 60 & 60 & $1-150$ & 13 & 6 & $0-84$ & $<0.01$ & 0.03 \\
\hline Ammonia (ppm) & 0.4 & 0 & $0-5$ & 1.37 & 0 & $0-7$ & 0.02 & $\mathrm{NI}^{3}$ \\
\hline Mean barn temperature $\left({ }^{\circ} \mathrm{C}\right)$ & 22.8 & 22.8 & $18.2-28.5$ & 13.6 & 13.5 & $7.8-18.0$ & $<0.01$ & NI \\
\hline Maximal barn temperature $\left({ }^{\circ} \mathrm{C}\right)$ & 23.2 & 23.2 & $18.5-29.5$ & 13.9 & 14 & $8-18.2$ & $<0.01$ & NI \\
\hline Mean relative hygrometry (\%) & 59.6 & 59.3 & $47.9-71.6$ & 26.9 & 26.5 & $18.7-40.1$ & $<0.01$ & NI \\
\hline Maximal relative hygrometry $(\%)$ & 61.2 & 60.6 & $50.1-74.0$ & 27.3 & 26.7 & $19.0-40.5$ & $<0.01$ & NI \\
\hline
\end{tabular}

${ }^{1} P$-values for comparing winter and summer environmental characteristics using a nonparametric Wilcoxon rank sum test for paired sample (winter vs. summer).

${ }^{2} P$-value resulting for univariable analysis. The counts were log-transformed and their association was tested in relation with prevalence of ultrasonographic lung consolidation ( $\geq 3 \mathrm{~cm}$ depth) using the event/trial syntax of the GLIMMIX procedure of SAS (version 9.4, SAS Institute Inc., Cary, NC).

${ }^{3} \mathrm{NI}$ (not included in the univariable analyses): these variables were not included in univariable analysis because of low variability observed (ammonia) or high correlation with season (temperature, hygrometry) $\geq 0.7$.

if no sign of overdispersion was observed, so long as the conditional distribution of Pearson chi-squared or degrees of freedom was close to 1 (Stroup, 2012). The least squares means were calculated for all significant variables in the final models and tested with TukeyKramer test.

Table 2. Univariable association of various herd-level characteristics and the proportion of ultrasonographic consolidation (defined as $\geq 3 \mathrm{~cm}$ depth) in preweaned calves

\begin{tabular}{|c|c|c|}
\hline Item & $\begin{array}{l}\text { Proportion }(\%) \text { of calves } \\
\text { with consolidation per herd } \\
\left(\mathrm{LSM} \pm \mathrm{SEM}^{1}\right)\end{array}$ & $P$-value ${ }^{2}$ \\
\hline \multicolumn{3}{|l|}{ Season } \\
\hline Summer & $8.9 \pm 2.1$ & $<0.01$ \\
\hline Winter & $17.4 \pm 3.5$ & \\
\hline \multicolumn{3}{|l|}{ Feeding } \\
\hline No automated milk feeder & $10.3 \pm 2.8$ & 0.73 \\
\hline Automated milk feeder & $12.4 \pm 5.5$ & \\
\hline Maximal milk allowance $<8 \mathrm{~L}$ & $11.51 \pm 4.9$ & 0.86 \\
\hline Maximal milk allowance $\geq 8 \mathrm{~L}$ & $10.5 \pm 2.9$ & \\
\hline Milk replacer & $10.9 \pm 2.8$ & 0.93 \\
\hline Whole milk \pm milk replacer & $10.3 \pm 5.5$ & \\
\hline All calves have water access & $12.7 \pm 4.6$ & 0.57 \\
\hline No water access or limited water access & $9.9 \pm 2.8$ & \\
\hline \multicolumn{3}{|l|}{ Housing and management } \\
\hline Individual housing until weaning & $6.7 \pm 2.1$ & $<0.01$ \\
\hline Group housing before weaning & $19.5 \pm 4.8$ & \\
\hline No direct or indirect contact with older animals & $9.2 \pm 3.0$ & 0.21 \\
\hline Direct or indirect contact with heifers & $20.8 \pm 7.7$ & \\
\hline Direct or indirect contact with adults & $8.9 \pm 3.5$ & \\
\hline Vaccination of preweaned calves for $\mathrm{BRD}^{3}$ & $16.2 \pm 4.3$ & 0.25 \\
\hline No vaccination of preweaned calves for $\mathrm{BRD}$ & $10.8 \pm 2.7$ & \\
\hline Air draft $<0.3 \mathrm{~m} / \mathrm{s}$ during the visit & $14.5 \pm 3.0$ & 0.08 \\
\hline Air draft $\geq 0.3 \mathrm{~m} / \mathrm{s}$ during the visit & $8.3 \pm 2.8$ & \\
\hline No perceived BRD problem during preweaning & $11.2 \pm 2.2$ & 0.05 \\
\hline Perceived BRD problem during preweaning & $19.4 \pm 4.3$ & \\
\hline
\end{tabular}




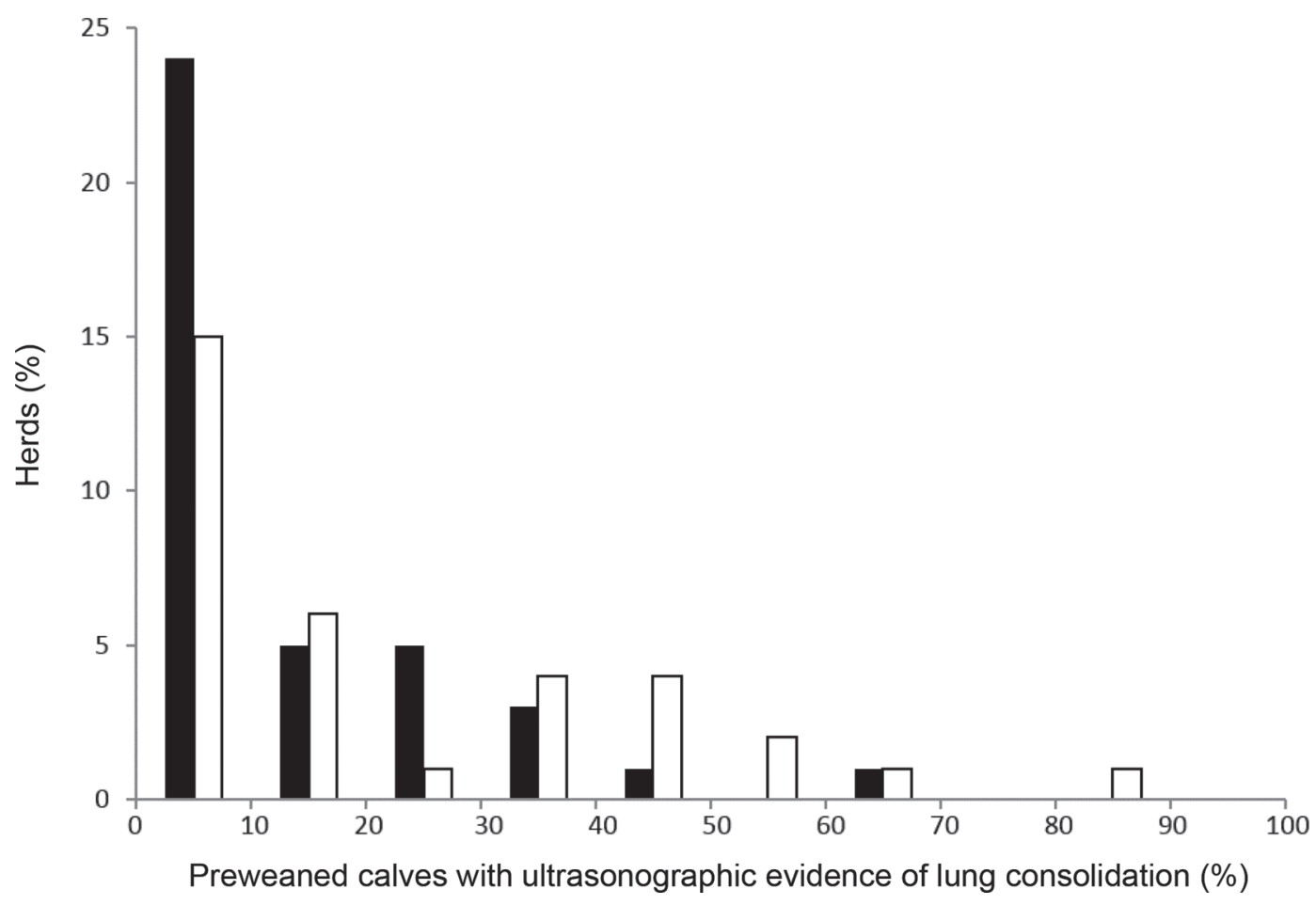

Figure 1. Seasonal distribution of the prevalence of preweaned calves with lung consolidation at the herd level. The distribution of proportion of consolidated calves (consolidation defined as at least 1 site of consolidation $>3 \mathrm{~cm}$ ) in 39 dairy herds during summer (black) and winter (white) season is represented. The prevalence of consolidation was higher in winter than in summer $(P<0.01$; see details in Table 2).

\section{RESULTS}

A total of 39 farms participated in the study, representing a $24.2 \%$ participation rate from the source population of herds $(\mathrm{n}=161)$. These farms had an average of 104 milking cows (median $=80$ cows; range $=55-280$ cows). Thirty-eight herds (97.4\%) used calf age as the sole criterion for weaning. Twenty herds (51.3\%) used group housing during the preweaning period and 8 (20.5\%) used an automated milk feeder. The maximal volume of milk or milk replacer that was allowed during preweaning varied greatly from a farm to another (median $=8 \mathrm{~L}$, range $=4-16 \mathrm{~L}$ ). The majority of farms ( $\mathrm{n}=32 ; 82.1 \%)$ used milk replacer, whereas some used raw milk or wasted milk for calf feeding (n $=7 ; 17.9 \%$ ). Water access was available for all calves in 25 farms (64\%). In 14 farms (36\%), all calves had no direct contact with heifers or cows, whereas in 8 farms $(21 \%)$ calves had a contact with heifers; in the 11 remaining farms a contact with adult was also possible. The percentage of farms where the owner perceived $\mathrm{BRD}$ as a problem were 23 and $26 \%$ for summer and winter, respectively. Eleven of 39 farms (28.2\%) used vaccination for BRD pathogens in preweaning calves, whereas 38 herds used cow vaccination against respiratory viruses (including bovine respiratory syncytial virus, bovine herpesvirus type 1 , bovine viral diarrhea virus, and parainfluenza 3 virus).

A total of 608 calves were enrolled during the entire study (2 sampling periods for each herd): 319 calves (median $=8$ per herd) during summer and 289 calves (median $=7$ per herd) during winter samplings. Only 9 herds $(23 \%)$ had 12 or more preweaning calves available at the time of the visit (12 to 27 available preweaning calves). In the remaining herds, all present preweaning calves were therefore included. The 50th percentile of the median age of sampled calves per herd was $38 \mathrm{~d}$ in summer [interquartile range $(\mathbf{I Q R})=28-50 \mathrm{~d}$ ] and 44 $\mathrm{d}$ in winter $(\mathrm{IQR}=33-50 \mathrm{~d})$. The median proportion of ultrasonographic consolidation was $8 \%$ (IQR $=0-22 \%$ ) in summer and $15 \%(\mathrm{IQR}=0-35 \%)$ in winter. The seasonal distribution is presented in Figure 1.

The Petrifilm plate counts [median (ranges) in cfu/ plate] were $365(90-1,001)$ for TAC, $4(1-35)$ for CC; and $12(0-300)$ for yeasts and $60(1-150)$ for molds in summer. During the winter, the TAC were 197.5 (4720), CC 1 (0-18), and yeasts 16.5 (1-310) and mold $6(0-84)$ and are summarized in Table 1 . We found a 
seasonal difference for TAC $(P<0.01)$, CC $(P<0.01)$, and molds $(P<0.01)$ but not for yeast counts $(P=$ $0.19)$.

The univariable statistical analysis results are summarized in Tables 2 and 3. Despite the fact that the probability of consolidation was significantly lower for calves in their first week of life when compared with calves in their fifth or more week (Figure 2), the age of calves in the herd was not associated with the odds of consolidation when controlling for herd effect $(P=$ $0.37)$. Two multivariable models were built including the farmer's perception of whether the herd had a problem with BRD or not (Table 3). In both models, the season and group housing during preweaning were associated with an increased odd of consolidation.

\section{DISCUSSION}

Thoracic ultrasound has recently gained in interest due to its potential for helping in BRD diagnosis (Ollivett and Buczinski, 2016). Our study found that the prevalence of lung lesions using thoracic ultrasound is relatively common in Québec dairy herds and is increased in herds with a perceived problem of BRD, herds that are group housed (vs. individually housed), and during winter season (vs. summer). These herdlevel risk factors have been previously associated with increased odds of respiratory disease or increased respiratory sounds when assessed by a veterinarian in a Swedish study (Lundborg et al., 2005). Unfortunately, and because of the wide variation of calf-raising systems in these relatively small farms, it was not possible to consistently assess the stocking density as a potential confounding factor for group-housed calves. Previous studies have shown that the size of the group rather than stocking density was associated with increased odds of respiratory disease at the calf level (Svensson et al., 2003).

Not surprisingly, our results showed that having a perceived BRD problem in preweaning calves was associated with an increased herd prevalence of ultrasonographic pulmonary consolidation. Producers' perception of BRD problems has not been extensively studied; however, perception of infectious disease may be affected by various factors (Frössling and Nöremark, 2016), including the accuracy of case detection. The diagnosis of enzootic pneumonia is a challenge in the absence of a reliable and accurate test. Application of clinical scoring charts, although appealing, may be difficult to implement in small farms on a regular basis (McGuirk, 2008; Love et al., 2014) due to the limited number of working people. Moreover, the sensitivity of producer detection or clinical scoring systems to detect $\mathrm{BRD}$ is generally lower than the specificity (Sivula et al., 1996; Love et al., 2016), which can explain why perception of a problem would be more related to the presence of a true problem (higher positive predictive value) than the opposite (i.e., not having a perceived BRD problem would not rule out a true BRD problem).

One of the limitations of thoracic ultrasound is that it is an indicator of cumulative incidence of BRD episodes for a particular calf. Indeed, when a calf faced a BRD episode, depending on the etiology (viral, bacterial, or mixed), lung lesions may appear as soon as a few hours, such as after an experimental challenge with Mannheimia haemolytica (Ollivett et al., 2013). However, little is known on the persistence of lung consolidation after BRD healing of an initial lung injury. For this reason, the proportion of calves with ultrasonographic lung lesions represent a mix of acute and chronic lung

Table 3. Effect of herd-level risk factors on the prevalence of ultrasonographic consolidation ( $\geq 3 \mathrm{~cm}$ depth) in preweaned calves

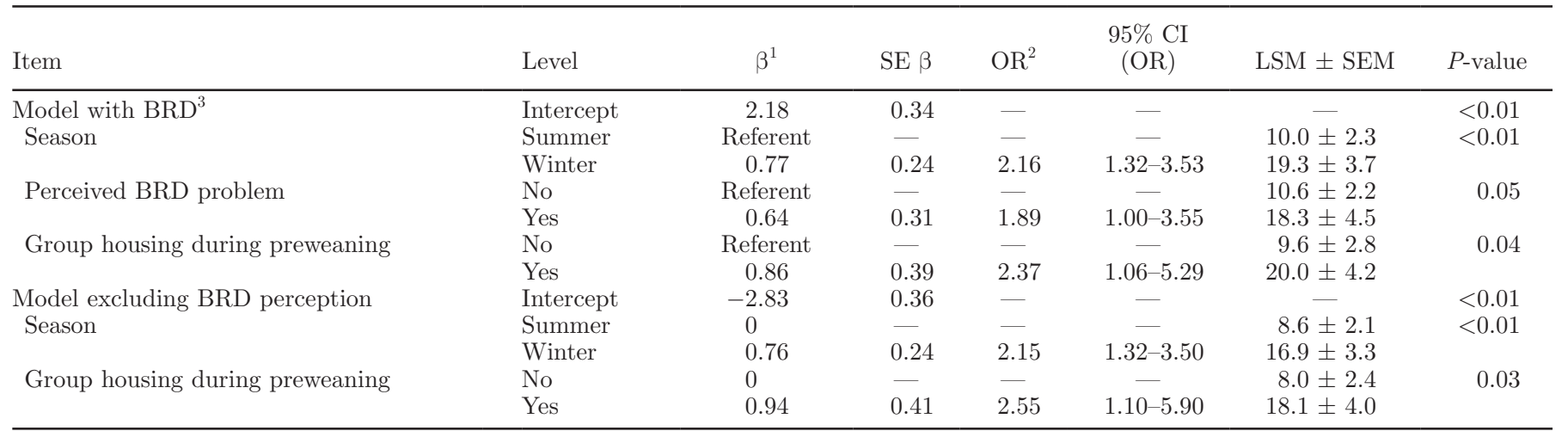

${ }^{1}$ Regression coefficient parameter.

${ }^{2}$ Odds ratio.

${ }^{3}$ Bovine respiratory disease complex. 


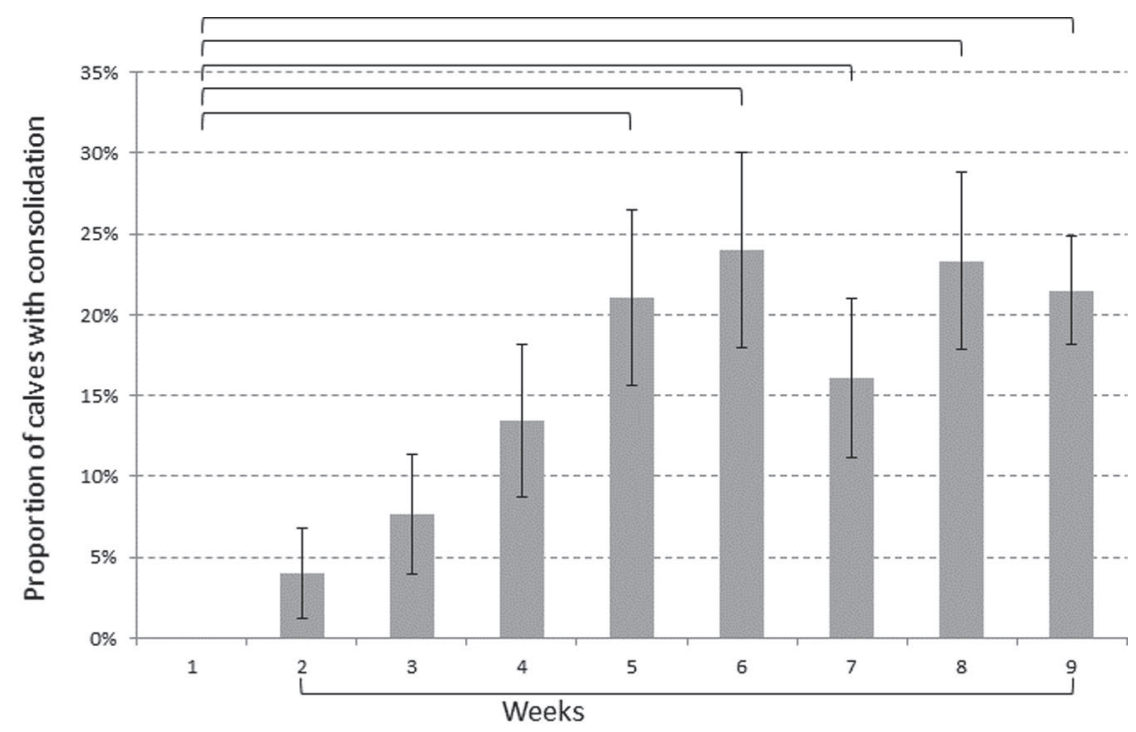

\begin{tabular}{|c|c|c|c|c|c|c|c|c|c|}
\hline Weeks & 1 & 2 & 3 & 4 & 5 & 6 & 7 & 8 & $\geq 9$ \\
\hline Calves with no consolidation & 37 & 48 & 48 & 45 & 45 & 38 & 47 & 46 & 117 \\
\hline Calves with consolidation & 0 & 2 & 4 & 7 & 12 & 12 & 9 & 14 & 32 \\
\hline Proportion of consolidation (\%) & 0 & 4 & 8 & 13 & 21 & 24 & 16 & 23 & 21 \\
\hline
\end{tabular}

Figure 2. Distribution of the proportion of calves with lung consolidation based on age stratification. The number of calves per week of age as well as the proportion ( $\pm \mathrm{SD}$ ) of calves with lung lesion (defined with a lesion of consolidation $\geq 3 \mathrm{~cm}$ ) are presented. The significant differences $(P<0.05)$ between weeks adjusted for multiple comparisons are presented using brackets.

lesions. No specific ultrasonographic findings have been reported to differentiate between acute and chronic lesions at this time.

At the calf level, it has been previously shown that extensive lung lesions at weaning have been associated with increased hazards of being culled before the first calving (Adams and Buczinski, 2016; Teixeira et al., 2017) and decreased reproduction efficiency after first calving (Teixeira et al., 2017). For these reasons, it is also of interest to accurately monitor lung lesions as a potential indicator of replacement heifer productivity. However, because decisions to change specific heifer management practices are made at the farm level, it was the objective of our study to be able to use thoracic ultrasound as a farm-level tool rather than as an individual test. An acceptable herd-level prevalence of consolidation threshold still needs to be established, but, based on the present study results, the first quartile of herds (i.e., $0 \%$ of calves with consolidation $>3$ $\mathrm{cm}$ ) would be a practical recommendation to define herds without pneumonia problems. In contrast, the last quartiles of herds (with consolidation proportion $>22 \%$ in summer and $35 \%$ in winter) could be a tentative reasonable guess for defining herds with abnormal proportions of consolidation. One of the limitations of our study was inherent to Québec dairy herd characteristics; as the herds are relatively small, it may be challenging to obtain a high number of calves to obtain a precise estimate of consolidation proportion in a limited period. From our study, a rule of thumb would be to have at least 6 calves with negative ultrasonographic examination to rule out a problem in winter [with a false-negative risk of $7.5 \%$ (1-0.35), with 0.35 defining the lower bound of the last quartile of winter prevalence, therefore assuming that every calf has the same probability of being consolidated] or 9 negative calves in summer [false-negative risk of $10 \%$ (1-0.22), with 0.22 defining the lower bound of the last quartile of summer prevalence]. Interestingly, despite the fact that very young calves (1st and 2 nd week of life) had lower probability of presenting lung lesions, the median age of sampled calves in the herd was not associated with the herd prevalence of consolidation when accounting for herd clustering; this suggests that herd-specific risk factors were more important than calf age distribution. Even if the median age of calves was not associated with the prevalence of consolidation in the present study, the probability of consolidation was low in calves less than 2 wk old, indicating that choosing calves $\geq 3$ wk old would increase the chances of finding lung consolidation. Care should therefore be taken extrapolating our findings, where the majority of calves are $<2$ wk old at time of ultrasound examination. 
It is also important to keep in mind that finding calves with lung lesions does not mean that lung consolidation should be used as a tool for treatment purpose. Studies in horse farms with enzootic Rhodococcus equi pulmonary infections have shown that relying only on lung lesions to treat foals increased antimicrobial use with no beneficial effect on foal health (Venner et al., 2013). Such information is currently missing in cattle.

We noted a seasonal effect on the prevalence of ultrasonographic lung consolidation, but we did not find any association between the calves' specific environment parameters and the prevalence of consolidation. Mold and coliform counts, as well as maximal air speed, were the only environmental variables that were selected during the univariable analysis, but they were partially confounded with season effect. As the environment assessment was done only once, and the observed consolidation could be related to a BRD episode that occurred days or weeks before the examination, the punctual measurement of environmental parameters (air speed, aerobic, coliform, YM counts, ammonia) may not reflect accurately the environmental conditions that the calves had before the day of data collection. Moreover, the limited numbers of herds may also be a limitation of the present study. Interestingly, ammonia levels were consistently low throughout our study and even lower than previously reported in winter (Seedorf and Hartung, 1999; Lago et al., 2006) or in summer (Kaufman et al., 2015). The inflammatory effect of high levels of ammonia on the respiratory tract has been mentioned in calves (Dewes and Goodall, 1995), but in a more recent Swedish study at the herd-level ammonia levels $<6 \mathrm{ppm}$ were counterintuitively associated with an increased odd of respiratory disease (odds ratio $=2.46$; 95\% CI $=1.16-4.93$; Lundborg et al., 2005). The association between this air quality parameter and BRD deserves future research.

In conclusion, our study showed that lung consolidation lesions detected by thoracic ultrasonography were present (median herd prevalence) in 8 and $15 \%$ of preweaning calves at the herd level in summer and winter, respectively. The prevalence of lung lesions was higher during winter and in farms that used group-housing during preweaning. Even if no specific herd-level prevalence threshold can be definitely proven from the present study, thoracic ultrasonography in preweaning dairy calves has potential to be used as a monitoring tool for respiratory health assessment in calves. From the present study, having no evidence of lung consolidation ( $\geq 3 \mathrm{~cm}$ lung consolidation) in up to 12 preweaning calves should be a goal for defining low-BRD risk farms. As BRD risks and herd dynamics or management are subject of change, assessment of ultrasonographic lung lesions should be done periodically at the herd level.

\section{ACKNOWLEDGMENTS}

The authors thank the Zoetis clinical research fund (bovine ambulatory clinic) of the Université de Montréal (St-Hyacinthe, QC, Canada) for partially financing the study as well as 3M Diagnostics (London, ON, Canada) for providing some of the Petrifilm used in that study for environment assessment. Parts of the material used in this study (anemometer, ammoniac detector) were obtained from a grant from the Fondation du Centre Hospitalier Universitaire Vétérinaire of the Université de Montréal (St-Hyacinthe, Québec, Canada). The authors also want to thank Clotilde Cros and Anne-Laure Lescarret (École Nationale Vétérinaire de Toulouse, France) for their valuable help in data collection during the summer part of the study and Jean-Philippe Pelletier (Faculté de Médecine Vétérinaire, St-Hyacinthe, Québec, Canada) for his valuable help during the winter sampling period.

\section{REFERENCES}

Adams, E. A., and S. Buczinski. 2016. Short communication: Ultrasonographic assessment of lung consolidation postweaning and survival to the first lactation in dairy heifers. J. Dairy Sci. 99:14651470 .

Aly, S. S., W. J. Love, D. R. Williams, T. W. Lehenbauer, A. Van Eenennaam, C. Drake, P. H. Kass, and T. B. Farver. 2014. Agreement between bovine respiratory disease scoring systems for preweaned dairy calves. Anim. Health Res. Rev. 15:148-150.

Beeler-Marfisi, J., M. E. Clark, X. Wen, W. Sears, L. Huber, C. Ackerley, L. Viel, and D. Bienzle. 2010. Experimental induction of recurrent airway obstruction with inhaled fungal spores, lipopolysaccharide, and silica microspheres in horses. Am. J. Vet. Res. 71:682-689.

Berman, J., D. Francoz, J. Dubuc, and S. Buczinski. 2017. A randomised clinical trial of a metaphylactic treatment with tildipirosin for bovine respiratory disease in veal calves. BMC Vet. Res. $13: 176$.

Blom, J. Y., E. Madsen, H. Krogh, and J. Wolstrup. 1984. Numbers of airborne bacteria and fungi in calf houses. Nord. Vet. Med. $36: 215-220$.

Buczinski, S., M. E. Borris, and J. Dubuc. 2017. Assessment of dairy calves' microbiological environment using Petrifilm bacteriology plates. Bov. Pract. 51:54-58.

Buczinski, S., G. Forté, D. Francoz, and A. M. Bélanger. 2014. Comparison of thoracic auscultation, clinical score, and ultrasonography as indicators of bovine respiratory disease in preweaned dairy calves. J. Vet. Intern. Med. 28:234-242.

Buczinski, S., T. L. Ollivett, and N. Dendukuri. 2015. Bayesian estimation of the accuracy of the calf respiratory scoring chart and ultrasonography for the diagnosis of bovine respiratory disease in pre-weaned dairy calves. Prev. Vet. Med. 119:227-231.

Cameron, M., G. P. Keefe, J. P. Roy, I. R. Dohoo, K. A. MacDonald, and S. L. McKenna. 2013. Evaluation of a 3M Petrifilm on-farm culture system for the detection of intramammary infection at the end of lactation. Prev. Vet. Med. 111:1-9.

Dewes, H. F., and G. Goodall. 1995. Some preliminary observations on the possible relationship between ammonia production from soiled bedding in calf rearing sheds and calf illness. N. Z. Vet. J. 43:37-41.

Frössling, J., and M. Nöremark. 2016. Differing perceptions-Swedish farmers' views of infectious disease control. Vet. Med. Sci. 2:54-68. 
Furman-Fratczak, K., A. Rzasa, and T. Stefaniak. 2011. The influence of colostral immunoglobulin concentration in heifer calves' serum on their health and growth. J. Dairy Sci. 94:5536-5543.

Gorden, P. J., and P. Plummer. 2010. Control, management, and prevention of bovine respiratory disease in dairy calves and cows. Vet. Clin. North Am. Food Anim. Pract. 26:243-259.

Guterbock, W. M. 2014. The impact of BRD: the current dairy experience. Anim. Health Res. Rev. 15:130-134.

Kaufman, J., M. Linington, V. Osborne, C. Wagner-Riddle, and T. Wright. 2015. Short communication: Field study of air ammonia concentrations in Ontario dairy calf housing microenvironments. Can. J. Anim. Sci. 95:539-542.

Khan, M. A., D. M. Weary, and M. A. von Keyserlingk. 2011. Invited review: Effects of milk ration on solid feed intake, weaning, and performance in dairy heifers. J. Dairy Sci. 94:1071-1081.

Lago, A., S. M. McGuirk, T. B. Bennett, N. B. Cook, and K. V. Nordlund. 2006. Calf respiratory disease and pen microenvironments in naturally ventilated calf barns in winter. J. Dairy Sci. 89:4014-4025.

Love, W. J., T. W. Lehenbauer, P. H. Kass, A. L. Van Eenennaam, and S. S. Aly. 2014. Development of a novel clinical scoring system for on-farm diagnosis of bovine respiratory disease in pre-weaned dairy calves. PeerJ 2:e238.

Love, W. J., T. W. Lehenbauer, A. L. Van Eenennaam, C. M. Drake, P. H. Kass, T. B. Farver, and S. S. Aly. 2016. Sensitivity and specificity of on-farm scoring systems and nasal culture to detect bovine respiratory disease complex in preweaned dairy calves. J. Vet. Diagn. Invest. 28:119-128.

Lundborg, G. K., E. C. Svensson, and P. A. Oltenacu. 2005. Herd-level risk factors for infectious diseases in Swedish dairy calves aged 0-90 days. Prev. Vet. Med. 68:123-143.

McGuirk, S. M. 2008. Disease management of dairy calves and heifers. T. Vet. Clin. North Am. Food Anim. Pract. 24:139-153.

McGuirk, S. M., and S. F. Peek. 2014. Timely diagnosis of dairy calf respiratory disease using a standardized scoring system. Anim. Health Res. Rev. 15:145-147.

Ollivett, T. 2014. Understanding the diagnosis and risk factors for respiratory disease in dairy calves. PhD Thesis, Population Medicine Department, University of Guelph, Guelph, Canada.

Ollivett, T., J. Hewson, R. Schubotz, and J. Caswell. 2013. Ultrasonographic progression of lung consolidation after experimental infection with Mannheimia haemolytica in Holstein calves. J. Vet. Intern. Med. 27:673.
Ollivett, T. L., and S. Buczinski. 2016. On-farm use of ultrasonography for bovine respiratory disease. Vet. Clin. North Am. Food Anim. Pract. 32:19-35.

Ollivett, T. L., J. L. Caswell, D. V. Nydam, T. Duffield, K. E. Leslie, J. Hewson, and D. Kelton. 2015. Thoracic ultrasonography and bronchoalveolar lavage fluid analysis in Holstein calves with subclinical lung lesions. J. Vet. Intern. Med. 29:1728-1734.

Pomés, A., M. D. Chapman, and S. Wunschmann. 2016. Indoor Allergens and Allergic Respiratory Disease. Curr. Allergy Asthma Rep. 16:43.

Pritchard, D. G., C. A. Carpenter, S. P. Morzaria, J. W. Harkness, M. S. Richards, and J. I. Brewer. 1981. Effect of air filtration on respiratory disease in intensively housed veal calves. Vet. Rec. 109:5-9

Seedorf, J., and J. Hartung. 1999. Survey of ammonia concentrations in livestock buildings. J. Agric. Sci. 133:433-437.

Sivula, N., T. Ames, W. Marsh, and R. Werdin. 1996. Descriptive epidemiology of morbidity and mortality in Minnesota dairy heifer calves. Prev. Vet. Med. 27:155-171.

Stroup, W. W. 2012. Generalized Linear Mixed Models: Modern Concepts, Methods and Applications. CRC Press, Boca Raton, FL.

Svensson, C., and P. Liberg. 2006. The effect of group size on health and growth rate of Swedish dairy calves housed in pens with automatic milk-feeders. Prev. Vet. Med. 73:43-53.

Svensson, C., K. Lundborg, U. Emanuelson, and S. O. Olsson. 2003. Morbidity in Swedish dairy calves from birth to 90 days of age and individual calf-level risk factors for infectious diseases. Prev. Vet. Med. 58:179-197.

Teixeira, A. G., J. A. McArt, and R. C. Bicalho. 2017. Thoracic ultrasound assessment of lung consolidation at weaning in Holstein dairy heifers: Reproductive performance and survival. J. Dairy Sci. 100:2985-2991.

Van Donkersgoed, J., C. S. Ribble, L. G. Boyer, and H. G. Townsend. 1993. Epidemiological study of enzootic pneumonia in dairy calves in Saskatchewan. Can. J. Vet. Res. 57:247-254.

Venner, M., K. Astheimer, M. Lammer, and S. Giguere. 2013. Efficacy of mass antimicrobial treatment of foals with subclinical pulmonary abscesses associated with Rhodococcus equi. J. Vet. Intern. Med. 27:171-176.

Windeyer, M. C., K. E. Leslie, S. M. Godden, D. C. Hodgins, K. D. Lissemore, and S. J. LeBlanc. 2014. Factors associated with morbidity, mortality, and growth of dairy heifer calves up to 3 months of age. Prev. Vet. Med. 113:231-240. 